\title{
ARTICLE OPEN \\ Controlling polar-toroidal multi-order states in twisted ferroelectric nanowires
}

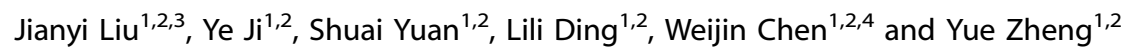

The toroidal order of electric dipoles in ferroelectric materials has attracted attention in the past decade due to fascinating properties and great potential for enabling novel memory devices, and functional devices in general. However, facile manipulation of toroidal order in ferroelectrics remains challenging. Here, using first-principles derived simulations, we demonstrate an efficient scheme to control the polar-toroidal multi-order (PTMO) states in ferroelectric nanowires. Two feasible strategies of controlling PTMO states by a combination of homogeneous electric field and torque are carried out in ferroelectric/paraelectric composite nanowires. This is possible based on trilinear coupling between polarization, toroidization and the twist force. As a result, switching of the toroidization of the nanowire can be readily achieved by reversal of the axial polarization. The torque threshold needed to control PTMO states is also calculated and found to be relatively small, indicating the feasibility of this method. Our study demonstrates facile control of PTMO states, including ferroelectric skyrmions, in ferroelectrics and is a step towards designing ferroelectric devices based on multi-order states.

npj Computational Materials (2018)4:78; https://doi.org/10.1038/s41524-018-0135-2

\section{INTRODUCTION}

Ferroelectrics are featured with a cooperative alignment of spontaneous electric dipoles, which can form fruitful dipole configurations (e.g., domain structures and topological states). Controllability of the dipole configurations modifies the material properties and is the basis of many device applications of ferroelectrics. ${ }^{1-3}$ For example, the switching of the polar state enables the creation of " 0 " and " 1 " states for binary functional devices design such as non-volatile memories and transistors. ${ }^{4}$ Diverse kinds of polar domain structures have been reported in ferroelectric thin films, e.g., stripe domain structure, ${ }^{5,6}$ ring-shaped domain structure, ${ }^{7,8}$ and zigzag domain structure, ${ }^{9,10}$ etc. Recently, it has been revealed that topological dipole states like vortices with a toroidal order ${ }^{11-13}$ can be stabilized in ferroelectric nanostructures under certain conditions. Interestingly, the toroidal order can coexist with polar order forming polar-toroidal multiorder (PTMO) states ${ }^{14,15}$ and even skyrmion-like states ${ }^{16}$ in specific ferroelectric nanostructures.

The intriguing topological dipole states, which can be stabilized in ferroelectrics with size being as small as several nanometers, have triggered intense interests due to their great promise for developing future functional devices. In the past decade, great efforts have been made in pursuit of switching the chirality of ferroelectric vortices by an experimentally feasible way. So far, the strategies of vortex switching reported in the literature are to introduce a region of "dominant dipoles" during vortex nucleation in ferroelectrics via different methods, such as making use of the geometry asymmetry, ${ }^{17}$ mechanical stress field, ${ }^{18,19}$ defect engineering, ${ }^{18-20}$ inhomogeneous electric field, ${ }^{21}$ and a sweeping biased tip. ${ }^{22}$ Despite these achievements, facile manipulation of the toroidal order in ferroelectrics remains challenging. In addition to the difficulty in experimental fabrication of high-quality ferroelectric vortex systems, one of the major reasons lies in the topological feature of ferroelectric vortices. On the one hand, the conjugate electric field of ferroelectric vortices is a curled field, which is difficult to realize in practice. On the other hand, the energy barrier between the vortex states with opposite chirality is usually large due to the topological protection.

More recently, PTMO states have come into focus for their potential in developing multi-state functional devices and the possibility of discovering ferroelectric skyrmion state. ${ }^{16,23}$ PTMO states have been theoretically predicted in several specific ferroelectric systems. A particular kind of systems are composite structures which consist of polar components and toroidal components like nanodots embedded in a polar matrix, ${ }^{24}$ and ferroelectric film-dot system. ${ }^{25}$ The second kind of systems are particular nanosystems made of rhombohedral phase ferroelectrics and under anisotropic screening condition, such as $\mathrm{BaTiO}_{3}$ and $\mathrm{BaTiO}_{3} / \mathrm{SrTiO}_{3}$ composite nanowires. ${ }^{14-16,23}$ The most important feature of a PTMO state is the coexistence and possible coupling between the polar order and toroidal order, which gives rise to the distinguished behaviors of a PTMO state compared with purely toroidal or polar state. For example, as a result of the polar-toroidal coupling, superior piezoelectric and piezotoroidal responses ${ }^{15}$ and interesting dynamical characteristics $^{26}$ are found in PTMO states. More importantly, we note that the coupling between polar order and toroidal order plays a significant role in dipole order transformation. ${ }^{15,27}$ Inspired by

\footnotetext{
${ }^{1}$ State Key Laboratory of Optoelectronic Materials and Technologies, School of Physics, Sun Yat-sen University, 510275 Guangzhou, China; ${ }^{2}$ Micro and Nano Physics and

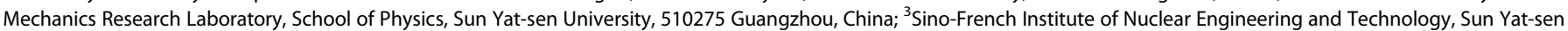
University, 519082 Zhuhai, China and ${ }^{4}$ School of Materials, Sun Yat-sen University, 510275 Guangzhou, China Correspondence: Weijin Chen (chenweijin@mail.sysu.edu.cn) or Yue Zheng (zhengy35@mail.sysu.edu.cn)
}

Received: 21 July 2018 Accepted: 22 November 2018

Published online: 18 December 2018 

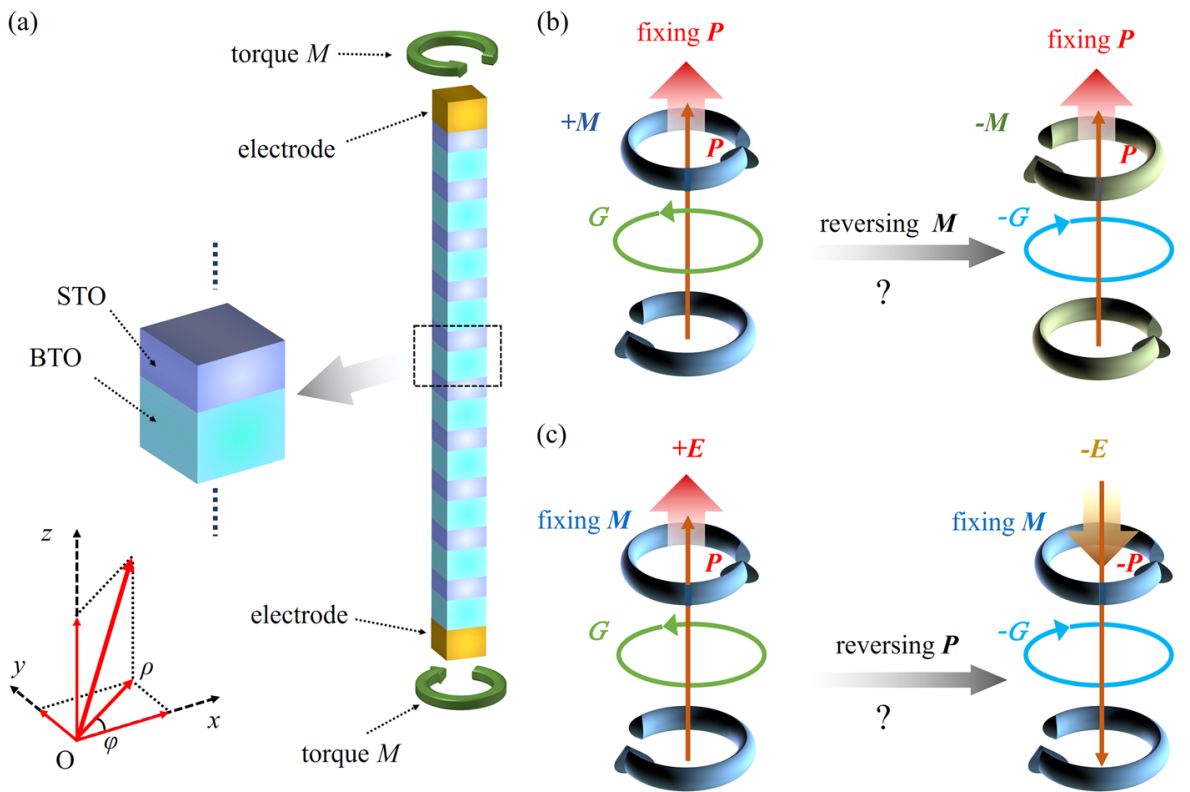

Fig. 1 Schematics of the model system and the basic idea of this investigation. a A periodic $\left(\mathrm{BaTiO}_{3}\right)_{m}\left(\mathrm{SrTiO}_{3}\right)_{n} \mathrm{Composite}$ nanowire. Two strategies of controlling PTMO states: b Chirality switching of PTMO state by switching torque $M$ and c chirality switching of PTMO state by switching the axial polarization $P_{\mathrm{z}}$

these results, it is highly expected that the polarity and chirality of a PTMO state can be facilely controlled based on the polartoroidal coupling.

This paper reports a novel control of the polarity and chirality of PTMO states in ferroelectric nanowires by twisting. Considering a ferroelectric nanowire as shown in Fig. 1a, the titled electric dipoles rotate around the $z$-axis and form a PTMO state. ${ }^{15}$ Assuming dipoles components along the $z$-axis are uniformly distributed and those perpendicular to $z$-axis are location dependent, increasing linearly with the increase of the distance with the center of the cross section. The maximum in-plane dipole component locating at the lateral surface is $p_{\mathrm{s}}$. The toroidization $G_{z}$ is given by $\mathbf{G}_{z}=\frac{1}{V} \sum_{i}^{N} \mathbf{r}_{i} \times \delta \mathbf{p}_{i}=A \frac{p_{s}}{a_{0}^{2}} \mathbf{e}_{z}$ (See Supplementary Information for more details), where $A$ is a constant depending on the geometry parameters of the nanowires, $V$ is the volume and $a_{0}$ is the lattice constant of the nanowire. Applying an external torque pair (with equal magnitude $M$ but in opposite sign at the top and bottom surfaces of the nanowire) to the nanowire induces shear stress $\tau_{z \varphi}$. The additional coupling energy caused by the coupling between the induced shear stress and the polarization components is $E^{\text {coup }}=-C P_{z} G_{z} \tau_{z \varphi}$ (See Supplementary Information for more details), with $C$ being a positive constant for a given nanowire. ${ }^{28}$ We can see that the torque induced shear stress $\tau_{z \varphi}$, toroidization $G_{z}$ and the axial polarization $P_{z}$ are coupling in a trilinear form. That is to say, if one fixes the axial polarization $P_{z}$ (polar order) of the nanowire, a change sign of shear stress $\tau_{z \varphi}$ (i.e., the torque $M$ ) will reverse the direction of the toroidization $G_{z}$ (toroidal order) of the nanowire (Fig. 1b). Moreover, fixing the shear stress $\tau_{z \varphi}$ acting on the nanowire, a change sign of the axial polarization $P_{z}$ can also lead to a reversal of the direction of toroidization $G_{z}$ (Fig. 1c). To verify the above argument, we carry out first-principles derived effective Hamiltonian simulations. The torque threshold needed to control PTMO states is relatively small, indicating high feasibility of this method. Our results reveal the potential of shear stress and strain in engineering exotic ferroelectric dipole states and provide a novel device design of ferroelectric multi-order states.

\section{RESULTS}

PTMO states in ferroelectric nanowires

$\left(\mathrm{BaTiO}_{3}\right)_{m}\left(\mathrm{SrTiO}_{3}\right)_{n}$ nanowires ( $m$ and $n$ refer to the thickness, in unit cells, of the (001) $\mathrm{BaTiO}_{3}$ and (001) $\mathrm{SrTiO}_{3}$ layers, respectively) adopt PTMO states due to the combining effect of the anisotropic electrostatic interaction of the nanowire, the $\mathrm{SrTiO}_{3}$-modified electrostatic interaction, and the rhombohedral ground state of $\mathrm{BaTiO}_{3} .{ }^{15}$ Here, using a first-principles derived effective Hamiltonian method combined with Monte Carlo (MC) simulations, we focus on $\left(\mathrm{BaTiO}_{3}\right)_{m}\left(\mathrm{SrTiO}_{3}\right)_{n}$ nanowires with the lateral size being $L_{x}=L_{y} \approx 4 \mathrm{~nm}$. The nanowires are simulated to cool down from a high temperature in the paraelectric region by a step of $10 \mathrm{~K}$. A random polarization perturbation is used to initiate the simulation, and it is used as the initial dipole state for the next temperature step until the stable dipole state is not paraelectric. By performing such an annealing process, the equilibrium dipole states of the nanowire at various temperatures can be obtained. Figure $2 a-c$ shows the phase diagram and corresponding dipole configurations of $\left(\mathrm{BaTiO}_{3}\right)_{10}\left(\mathrm{SrTiO}_{3}\right)_{2}$ and pure $\mathrm{BaTiO}_{3}$ nanowires. One can see that the two nanowires both adopt a pure polar state with nonzero axial polarization but zero toroidization at higher temperature, and they adopt a PTMO state with nonzero axial polarization and toroidization at lower temperature. The presence of $\mathrm{SrTiO}_{3}$-layer mainly modifies the electrostatic interaction, giving rise to the difference in the phase transition temperature and the stability range of polar state and the PTMO state. In the following, we will take $\left(\mathrm{BaTiO}_{3}\right)_{10}\left(\mathrm{SrTiO}_{3}\right)_{2}$ nanowire as an example, which exhibits a stable PTMO state when the temperature is below $200 \mathrm{~K}$. Similar results are also expected in the pure $\mathrm{BaTiO}_{3}$ nanowire and other $\left(\mathrm{BaTiO}_{3}\right)_{m}\left(\mathrm{SrTiO}_{3}\right)_{n}$ nanowires.

As shown in Fig. 1, we apply an external torque pair along the axial direction to twist the $\left(\mathrm{BaTiO}_{3}\right)_{10}\left(\mathrm{SrTiO}_{3}\right)_{2}$ nanowire. For simplicity, here the elastic properties of the two components are taken to be the same. In the simulations, the application of torque to the nanowire is realized by taking into account the twisting induced strain of this nanowire, which can be obtained by solving the mechanical equilibrium equation with appropriate boundary conditions using a finite element method. Readers are referred to Supplementary Information for more detailed discussion about the torque application to the nanowire. The torque pair creates a 
(a)

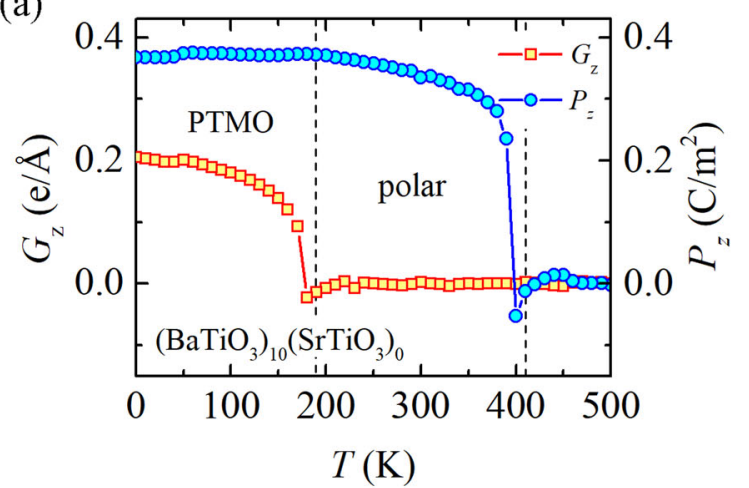

(c)

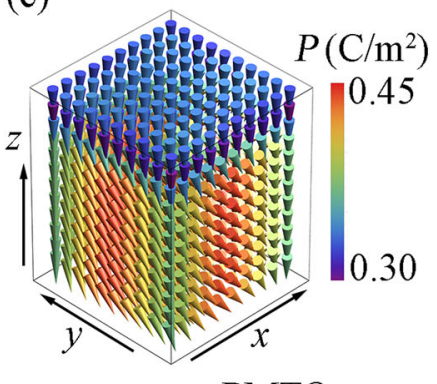

PMTO state

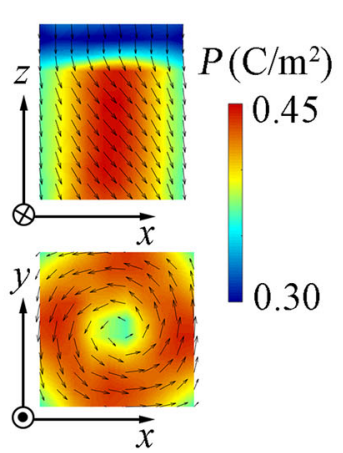

(b)

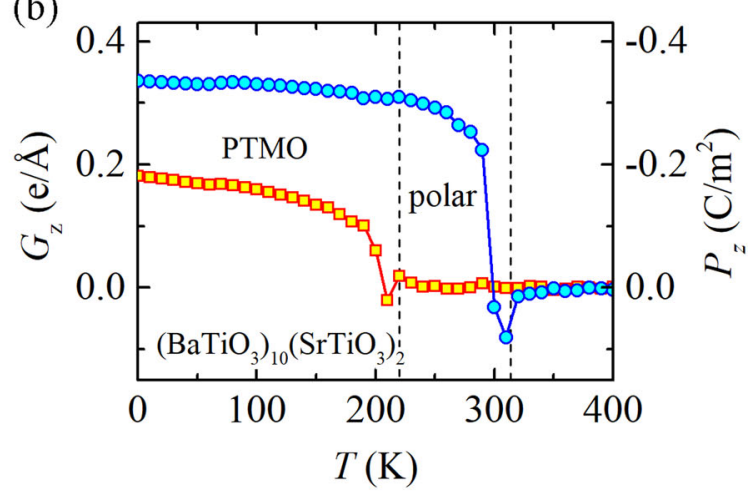

(d)

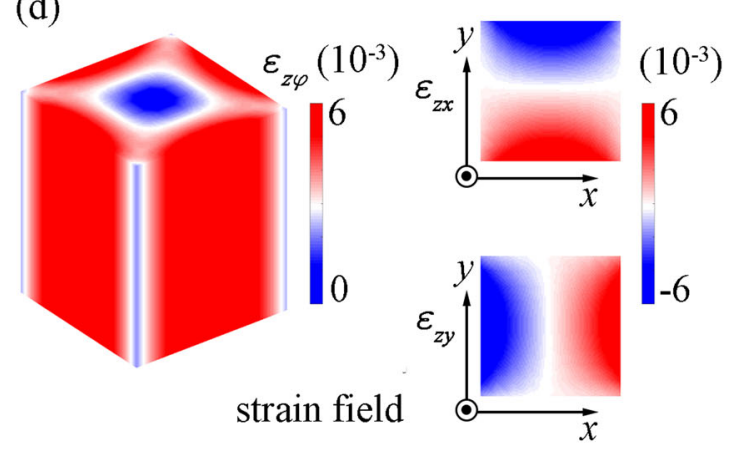

Fig. 2 Dipole states of nanowires during a cooling-down process. Evolution of toroidization and polarization in a $\mathrm{BaTiO}_{3}$ and $\mathbf{b}\left(\mathrm{BaTiO}_{3}\right)_{10} /$ $\left(\mathrm{SrTiO}_{3}\right)_{2}$ nanowires. c The dipole configurations of PTMO state in $\left(\mathrm{BaTiO}_{3}\right)_{10} /\left(\mathrm{SrTiO}_{3}\right)_{2}$ nanowires. $\mathbf{d}$ The distribution of the strain field caused by an external torque pair of about $100 \mathrm{nN} \mathrm{nm}$

distribution of shear stress and strain in the nanowire. Figure $2 d$ shows the distribution of the strain field in the nanowire under an external torque pair being about $100 \mathrm{nN} \mathrm{nm}$. One can see that the shear strain on the cross section of the nanowire $\varepsilon_{z \varphi}$ gradually increases along radial direction. Moreover, $\varepsilon_{z x}$ and $\varepsilon_{z y}$ defined in Cartesian coordinate system take maximum at the $x$ and $y$ surface of the nanowire, respectively, and they distribute asymmetrically with respect to the axis of the nanowire. Note that the warping displacement caused by the twisting is quite small, being on the order of pm (See Supplementary Information Fig. S2). Therefore, we suppose that the square cross sections keep plane during the deformation (As shown in Fig. 2d), which should not change the physics in this work.

Strategy 1: chirality switching of PTMO state by torque. Now we take the PTMO state formed in the $\left(\mathrm{BaTiO}_{3}\right)_{10}\left(\mathrm{SrTiO}_{3}\right)_{2}$ nanowire at $150 \mathrm{~K}$ as an example. The specific PTMO state possesses an axial polarization $P_{z}=-0.32 \mathrm{C} / \mathrm{m}^{2}\left(P_{z}<0\right)$ and an axial toroidization $G_{z}=0.14 e / \AA\left(G_{z}>0\right.$, a counterclockwise (CCW) vortex) as depicted in Fig. 2c. The nanowire is twisted by an applied torque pair (180 nN nm, CCW at the top surface of the simulation cell) while keeping the direction of the axial polarization fixed $\left(P_{z}<0\right)$. The MC simulated evolution of the PTMO state is shown in Fig. $3 \mathrm{a}_{\text {, }}$ c. We can see that the direction of the toroidization $G_{z}$ is reversed after about $70,000 \mathrm{MC}$ sweeps while the axial polarization $P_{z}$ remains unaffected during the whole process. Moreover, it is noteworthy that the polar state in the nanowire is quite stable, no additional electric field is needed to fix the direction of the axial polarization during the switching process in this case. This is an important feature caused by the coexistence of polar and toroidal orders of PTMO state. Despite this, the application of an external electric field is quite helpful to reduce the threshold of the torque needed to switch the chirality of the toroidal order (i.e., the coercive torque, $M_{C}$ ) and the corresponding relaxation time, which is discussed in detail later. Figure $3 c$ depicts the evolution of the dipole configuration during the switching process, which varies from the initial state $A\left(P_{z}<0, G_{z}>0\right)$ to an intermediate state $B$ $\left(P_{z}<0, G_{z} \sim 0\right)$ and finally stabilizes at the final state $C\left(P_{z}<0, G_{z}\right.$ $<0$ ), which is a degenerate state of state $A$. Similar results can also be obtained for cases with $P_{z}>0$.

We further investigate the dependence of $G_{z}$ and $P_{z}$ of the evolved PTMO state on the magnitude of the torque at $150 \mathrm{~K}$. To achieve this, a sequence of torque pair with its value varying in a triangle wave form is applied to the nanowire and the system is fully relaxed at every applied torque pair. Such an application of torque pair will result a hysteresis loop of $G_{z}$ as a function of $M$, as shown in Fig. 3b. The coercive torque $M_{C}$ is about $150 \mathrm{nN} \mathrm{nm}$ and the remnant toroidization $G_{R}$ is approximately $0.14 e / \AA$. This clearly verifies that there is indeed an intrinsic coupling between the toroidal order of the PTMO state and the torque induced cross-sectional shear stress. Moreover, $P_{z}$ keeps unchanged during the process as expected, showing that axial polar order is independent of twisting due to the relatively small torque in this case.

The relationship between $G_{z}$ and $M$ at various temperatures is also investigated and the result is plotted in Fig. 4a (See Supplementary Information Fig. S3 for more details). We obtain similar hysteresis loops at different temperatures, but the values of $M_{C}$ and $G_{R}$ are strong functions of temperature, which are further summarized in Fig. $4 \mathrm{~b}$. It is shown that $M_{C}$ and $G_{R}$ decrease rapidly with increasing temperature. Moreover, we note that the shape of the two curves are similar, indicating that $M_{C}$ is mainly determined by $G_{R}$ when there are no other loads. This is easy to be understood by considering the fact that, at higher temperature, the polarization magnitude is smaller and the nanowire tends to adopt an axial polar state (See, Fig. 2). As a consequence, both the toroidization (determined by the magnitude of the in-plane polarization) and the energy barrier of chirality switching (which 


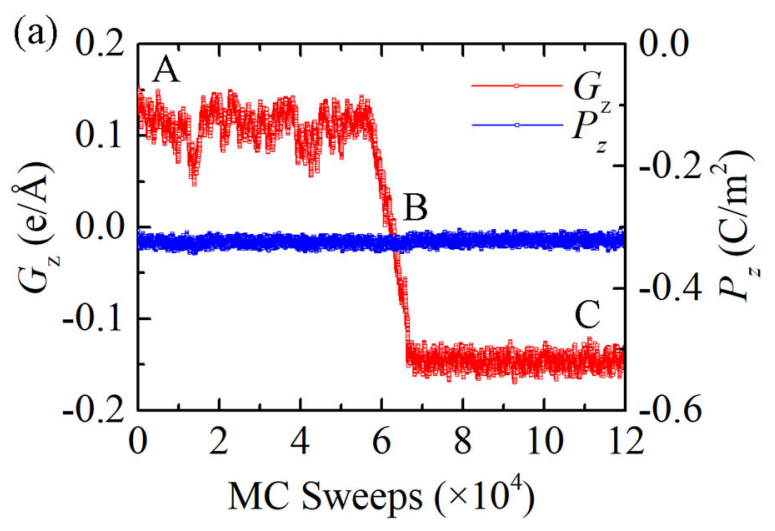

(c) $\mathrm{A}$

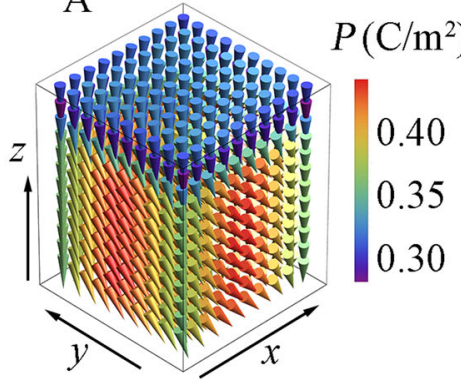

initial state $\left(-P_{Z},+G_{Z}\right)$

B

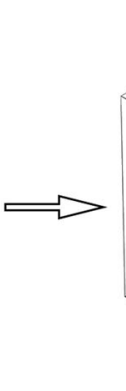

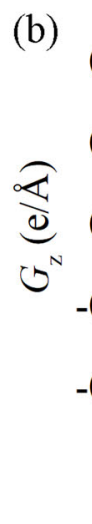

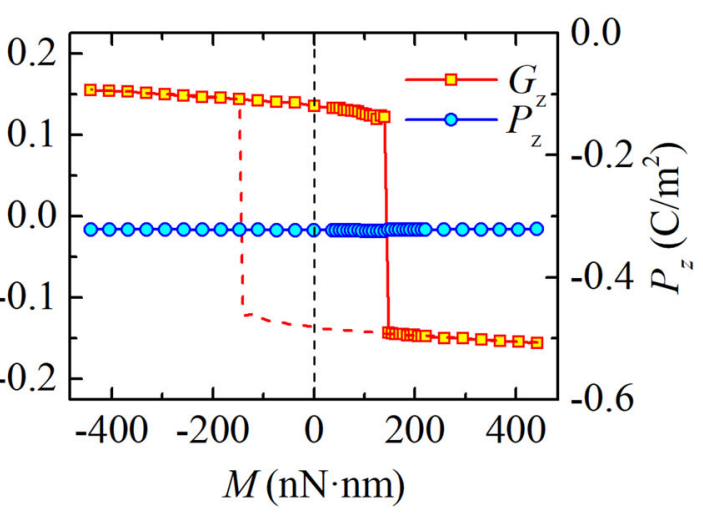

intermediate state $\left(-P_{z}, G_{z} \sim 0\right)$
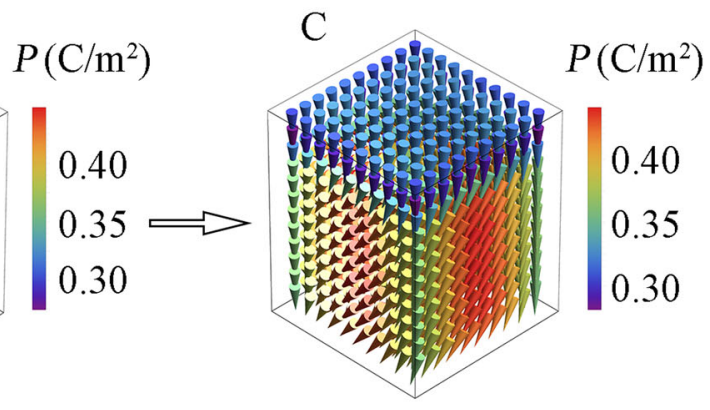

final state $\left(-P_{z},-G_{z}\right)$

Fig. 3 Chirality switching of PTMO state by torque. Evolution of the PTMO state of a $\left(\mathrm{BaTiO}_{3}\right)_{10}(\mathrm{SrTiO})_{2}$ nanowire under a counterclockwise torque pair at $T=150 \mathrm{~K}$. a Evolution of the toroidization $G_{z}$ and axial polarization $P_{z}$ with the torque $M$ being $180 \mathrm{nN} \mathrm{nm}$. b The toroidization $G_{z}$ and axial polarization $P_{z}$ of the PTMO state as functions of torque $M$ at $150 \mathrm{~K}$. c The corresponding evolved dipole configurations during the switching process

must be overwhelmed by the trilinear coupling energy $E_{\mathrm{C}}$ to achieve switching) decrease when temperature increases, leading to a decrease of $G_{R}$ and $M_{C}$. Such a temperature controllability of chirality switching of PTMO state should be useful in practical characterization and applications.

Strategy 2: chirality switching of PTMO state by switching the axial polarization. The chirality of the toroidal order can also be switched by reversing the direction of the polarization. This is achieved by applying an external homogeneous electric field along the $z$-axis while the torque acting on the nanowire is fixed at the same time. Again, we take the PTMO state of the $\left(\mathrm{BaTiO}_{3}\right)_{10}\left(\mathrm{SrTiO}_{3}\right)_{2}$ nanowire at $150 \mathrm{~K}$ as an example. As shown in Fig. $5 \mathrm{a}$, the nanowire is twisted by an external torque pair of $70 \mathrm{nN} \mathrm{nm}$ (clockwise (CW) at the top surface of the simulated cell). An electric field of $1.5 \mathrm{MV} / \mathrm{cm}$ along the $+z$ direction is then applied to reverse the direction of the polarization at the 10,000th MC sweep. We can see from inset of Fig. 5 a that $P_{z}$ reverses very quickly and changes its sign within $400 \mathrm{MC}$ sweeps, and immediately the reversal of the direction of $G_{z}$ occurs after about $4000 \mathrm{MC}$ sweeps. The external electric field is removed at the 40,000th MC sweep, which results in a sudden drop of the polarization and increase of the toroidization in Fig. 5a. In particular, note that the switching process here is much faster than that in the previous strategy. We attribute this difference to the application of the external electric field. The response of polar order to electric field is much faster than that of the toroidal order to mechanical loads. More importantly, the electric field directed along $z$-axis acting on the nanowire enhances the polar order and weakens the toroidal order of the PTMO state after polarization reversal, leading to the reduction of the switching barrier of the chirality. As a result, the chirality of the toroidal order can be switched in a much easier and quicker way in this case. The corresponding dipole configurations during the switching process are depicted in Fig. 5c. Specifically, when a reversed electric field is applied to the initial PTMO state, i.e., state $A\left(P_{z}<0, G_{z}>0\right)$, the axial polarization $P_{z}$ is firstly decreased quickly while the toroidization $G_{z}$ is enhanced, forming an intermediate state $B$ $\left(P_{z} \sim 0, G_{z}>0\right)$. Immediately, $P_{z}$ is reversed and further increased to a large value, and $G_{z}$ is decreased at the same time as shown in state $C\left(P_{z}>0, G_{z}>0\right)$. Then, $G_{z}$ gradually reduces to zero and is reversed as shown in state $D\left(P_{z}>0, G_{z}<0\right)$, further forming state $E$ $\left(P_{z}>0, G_{z}<0\right)$ after enough time. After removal of the external electric field, the nanowire stabilizes at the final PTMO state $\mathrm{F}\left(P_{z}\right.$ $\left.>0, G_{z}<0\right)$, which is a degenerate state of state $A$. Similarly, this switching strategy also works for cases with $P_{z}>0$.

The dependence of $G_{z}$ and $P_{z}$ of the PTMO state on the magnitude of electric field under a fixed torque pair of $70 \mathrm{nN} \mathrm{nm}$ (CW at the top surface of the simulated cell) at $150 \mathrm{~K}$ is further investigated and the result is shown in Fig. 5b. A sequence of electric field with its value varying in a triangle wave form is applied on the nanowire along $z$-axis and the system is fully relaxed at every applied electric field. The important results are as follows: (1) The relationship between $P_{z}$ and applied electric field $E$ is a typical ferroelectric hysteresis loop. The remnant polarization $P_{R}$ is about $0.32 \mathrm{C} / \mathrm{m}^{2}$ and the coercive electric field $E_{\mathrm{C}}$ is approximately $0.5 \mathrm{MV} /$ $\mathrm{cm}$. This hysteresis loop is similar to our previous simulated results obtained without mechanical loads. ${ }^{15}$ This reflects that fact that the torque does not significantly influence the intrinsic coupling between the polar order and electric field along z-axis, as the axial polarization is strong and almost unaffected by the torque employed in the simulation at $150 \mathrm{~K}$. (2) The relationship between $G_{z}$ and $E$ is also a hysteresis loop. The remnant toroidization $G_{R}$ is $0.14 \mathrm{e} / \AA$ and the coercive electric field $E_{\mathrm{C}}$ is about $0.7 \mathrm{MV} / \mathrm{cm}$. Note 
(a)

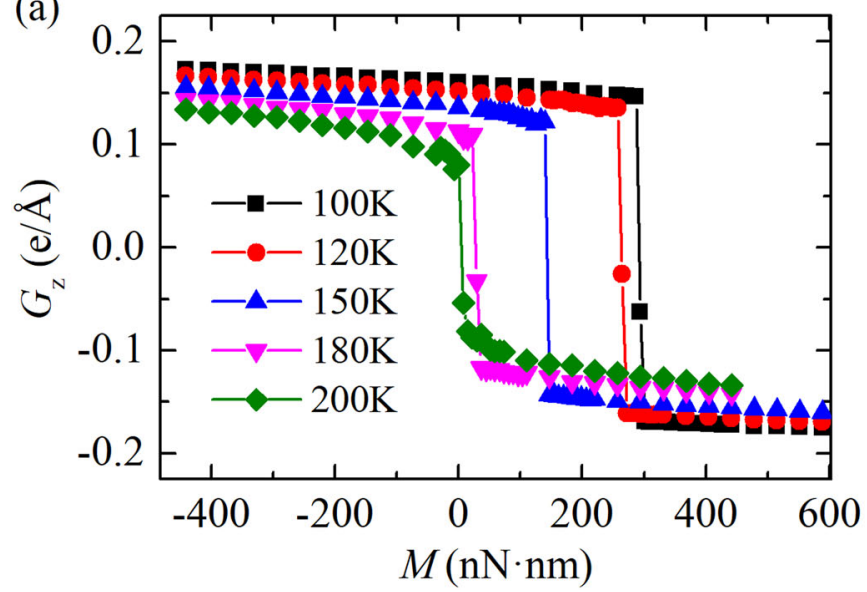

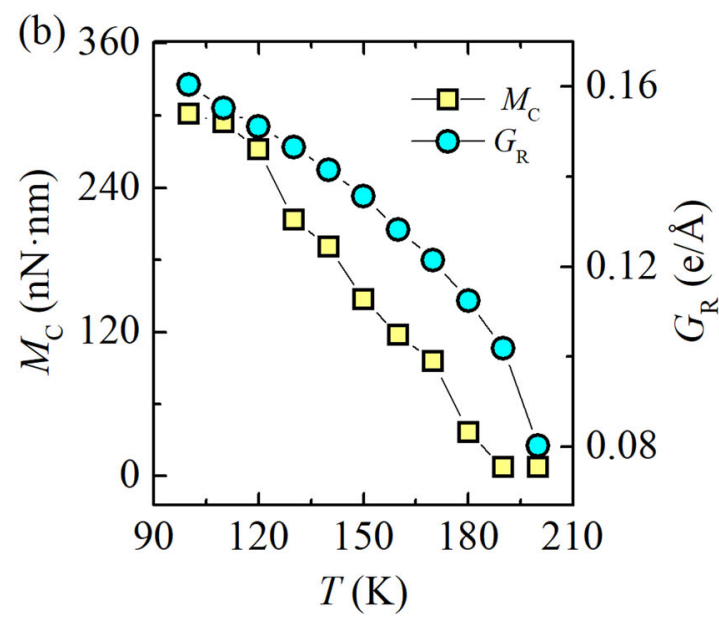

Fig. 4 Temperature dependence of the controllability of PTMO state by torque. a The calculated toroidization-torque $\left(G_{z}-M\right)$ hysteresis loops and $\mathbf{b}$ corresponding coercive torque $M_{\mathrm{C}}$ and remnant toroidization $G_{\mathrm{R}}$ of a $\left(\mathrm{BaTiO}_{3}\right)_{10}\left(\mathrm{SrTiO}_{3}\right)_{2}$ nanowire at different temperatures

that the shape of the hysteresis loop of $G_{z}-E$ is different with those of traditional ferroelectric hysteresis loops. This is due to the combination effect of the applied torque and electric field. Specifically, when the external electric field and the polar order is in opposite direction and the magnitude of the electric field is small $\left(E<E_{C}\right)$, the sign of the $P_{z}$ remains fixed and the magnitude of the $P_{z}$ is significantly reduced. The dipoles of the nanowire favor aligning along the in-plane direction (perpendicular to $z$-axis), indicating that the polar order is weakened and the toroidal order is enhanced. As a result, the sign of $G_{z}$ keeps unchanged and the magnitude of $G_{z}$ increases with the reversal electric field. However, when $E>E_{C}$, both the sign of the $P_{z}$ and $G_{z}$ are changed, and the magnitude of the polarization increases. As the dipoles of the nanowire favor aligning along the axial direction, the polar order is enhanced and the toroidal order is weakened. Thus, the magnitude of $G_{z}$ decreases with the increase of the applied electric field. $G_{z}$ tends to zero when the magnitude of electric field becomes very large $\left(E>E_{C}\right)$. The PTMO state approaches a purely polar state along the axial direction with the cross-sectional toroidal order nearly vanished.

For this strategy of chirality switching of the PTMO state, we further investigate the relationship between $G_{z}$ and $E$ at various temperatures and torques. Figure 6 a shows the $G_{z}-E$ hysteresis loops at various temperatures under a fixed torque pair of $70 \mathrm{nN}$ $\mathrm{nm}$ (CW at the top surface of the simulated cell). The remnant toroidization $G_{\mathrm{R}}$ and the coercive electric field $E_{\mathrm{C}}$ as functions of temperature and torque are summarized in Fig. 6b, c (See also Supplementary Information Fig. S4 for more details), respectively. One can see that the nanowire maintains a relatively large and stable $G_{R}$ at temperature $(\sim 200 \mathrm{~K})$ near the phase boundary between the PTMO state and pure polar state. $G_{R}$ is larger for larger torque, and the effect of torque on $G_{R}$ is more significant at higher temperature. Moreover, the coercive electric field $E_{C}$ is larger at lower temperature, and it is smaller for larger torque. The effect of torque on $E_{C}$ is more significant at lower temperature. This indicates that torque can largely affect the barrier of axial polarization switching at lower temperature, despite its relatively small effect on the toroidal order (Fig. 6b). Importantly, our results show that with the help of a torque pair as small as $37 \mathrm{nN} \mathrm{nm}$, the chirality of the PTMO state can be reversed by an axial external electric field, which is highly feasible in practice.

\section{DISCUSSION}

Let us review the additional free energy due to the coupling between the shear stress and polarization components. When the nanowire is twisted, the free energy difference is
$\Delta F=-A Q \tau_{z \phi} G_{z} P_{z}$. The condition $\Delta F<0$ must be satisfied from the viewpoint of thermodynamics to make a dipole state stable in the twisted nanowire. Since both the integration constant $A$ and electrostrictive coefficient $Q$ are positive, we must have $\tau_{z \phi} G_{z} P_{z}>$ 0 . Therefore, four PTMO states may exist stably in the twisted nanowire, i.e., A: $P_{z}<0, G_{z}>0, \tau_{z \varphi}<0$ (i.e., $M<0$ ); B: $P_{z}<0, G_{z}<0$, $\tau_{z \varphi}>0$; C: $P_{z}>0, G_{z}>0, \tau_{z \varphi}>0$; D: $P_{z}>0, G_{z}<0, \tau_{z \varphi}<0$. Accordingly, one can obtain four routes of switching the chirality of PTMO states: $A \rightleftarrows B$ and $C \rightleftarrows D$ by reversing the shear stress $\tau_{z \varphi}$ and keeping direction of the axial polarization unchanged, and $A \rightleftarrows D$ and $B \rightleftarrows C$ by switching the axial polarization and keeping the sign of shear stress $\tau_{z \varphi}$ unchanged.

We would like to make a comment on the experimental feasibility of the proposed strategies, regarding to the fabrication of systems carrying PTMO states and the loading of twist force on nanostructures. Experimental fabrication of high quality PTMO carriers in ferroelectric nanowires is a big challenge. A combination of physical vapor deposition (PVD) and stencil lithography should allow the preparation of ferroelectric composite nanostructures. $^{29,30}$ There are also publications reporting growing composition-modulated nanowires through the modulation of the seed particle size by tuning the supply of precursors during the growth, ${ }^{31}$ which is possibly applicable to the fabrication of ferroelectric composite nanowire systems. Besides, one can notice that the preparation process of $\mathrm{BaTiO}_{3}$ nanowire, ${ }^{32-35}$ which also have PTMO states, is mature in the literature. Moreover, PTMO states should not be limited to $\mathrm{BaTiO}_{3}$ nanowires. $\mathrm{BaTiO}_{3}$ nanodots under appropriate charge screening conditions are also promising systems to carry PTMO states (See Supplementary Information Fig. S5). Moreover, our idea can also be employed to switch the charity of the vortex domain structures in ferroelectric nanodots. However, note that the existence of a polar order is crucial during the switching by torque as discussed above. An additional electric field is needed to introduce a PTMO state in the ferroelectric nanodots to carry out our strategies. ${ }^{36}$ The PTMO state works as a transition state during the charily of the vortex domain structure switching in ferroelectric nanodots. Note also that skyrmion-like states, whose existence in ferroelectrics has recently been a particularly interesting issue, are actually PTMO states. Our strategies thus provide a facile control of the chirality of skyrmion-like states in ferroelectric systems. As for the loading of twist force on nanostructures, we would like to point out that twisting is a common phenomenon in nanostructures. A nanowire can be twisted during fabrication and manipulation due to various factors such as defects. ${ }^{37-39}$ However, exerting a well-controlled twist force on a nanowire in practice requires some tactics. There 

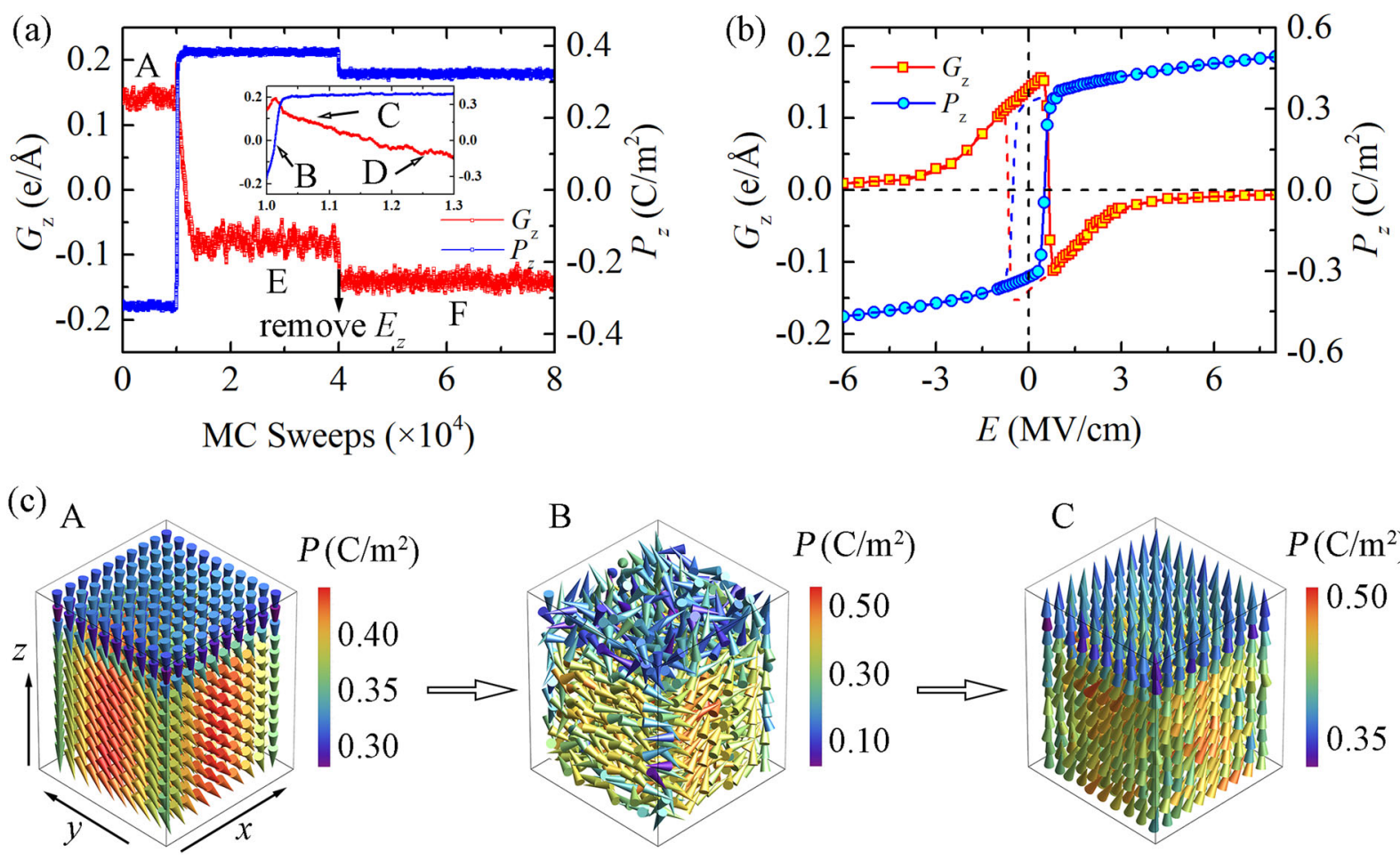

initial state $\left(-P_{z},+G_{z}\right)$
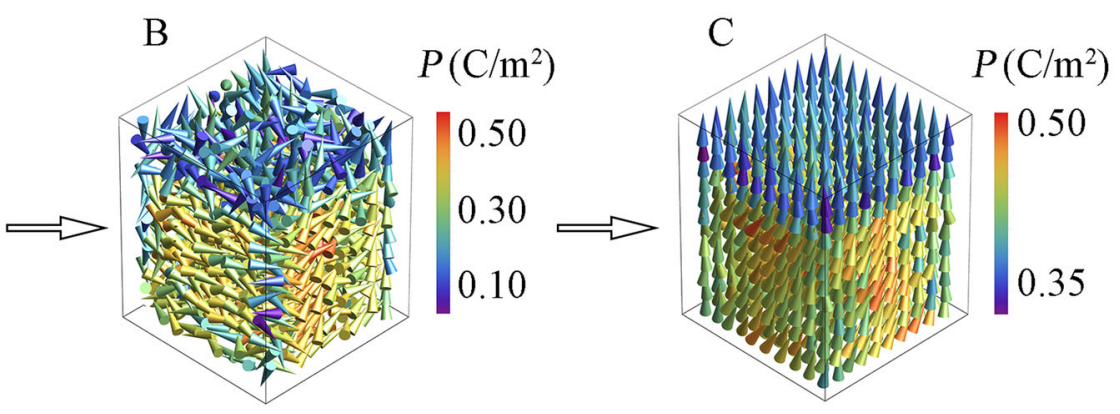

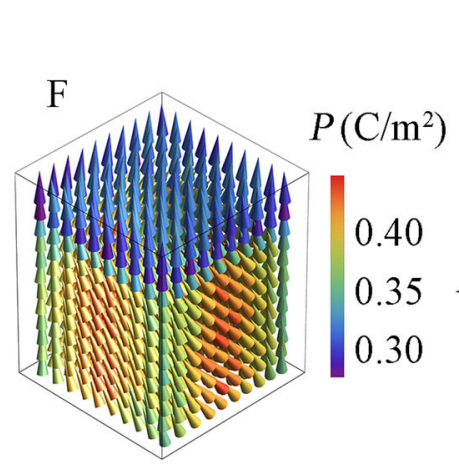

final state $\left(+P_{Z},-G_{Z}\right)$

intermediate state1 $\left(P_{Z} \sim 0,+G_{Z}\right) \quad$ intermediate state $2\left(+P_{Z},+G_{Z}\right)$

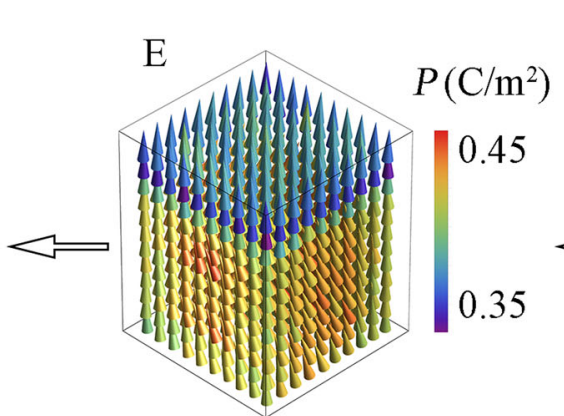

intermediate state $4\left(+P_{z^{3}}-G_{z}\right)$

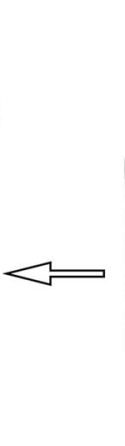

intermediate state3 $\left(+P_{Z},-G_{Z}\right)$

Fig. 5 Chirality switching of PTMO state by switching the axial polarization. Evolution of the PTMO state of a $\left(\mathrm{BaTiO}_{3}\right)_{10}\left(\mathrm{SrTiO}_{3}\right)_{2}$ nanowire under a $+z$ directed electric field and a clockwise torque pair of $70 \mathrm{nN} \mathrm{nm}$ at $T=150 \mathrm{~K}$. a Evolution of the toroidization $G_{z}$ and axial polarization $P_{z}$ with the electric field $E_{z}=1.5 \mathrm{MV} / \mathrm{cm}$. b The toroidization $G_{z}$ and axial polarization $P_{z}$ of the PTMO state as functions of electric fields $E$ at $150 \mathrm{~K}$. c The corresponding evolved dipole configurations during the switching process

are several methods for reference. ${ }^{40-44}$ One possible scheme is using a scanning probe microscopy (SPM) tip scanned over the lateral side of an epitaxial nanowire, thus a shear stress can be inducing due to the friction between the tip and the nanowire. ${ }^{40,41}$ Another scheme is fixing the two terminals of nanowires by designing particular clamp, such as zigs. ${ }^{42,43}$ Then the twist force can be directly acted on the nanowires by manipulation of the clamp. Besides, microfabricated gripper is also a usable tool providing good control over twist force on nanowires. ${ }^{44}$

In conclusion, first-principles derived simulations have been performed to reveal the controllability of PTMO states in $\left(\mathrm{BaTiO}_{3}\right)_{m}\left(\mathrm{SrTiO}_{3}\right)_{n}$ nanowires under twist force. We demonstrate two feasible strategies of controlling the chirality of PTMO state via a combined loading of axial electric field and twist force. The chirality switching of the PTMO state exploits the trilinear coupling relation between polarization, toroidization and the twist force.
Furthermore, our investigation shows that the PTMO state exhibits large $G_{R}$ and $P_{R}$, which can be regularly controlled by a relatively small electric field and torque. We finally discuss the reliability and feasibility of the proposed strategies. Our study verifies the controllability of exotic multi-order dipole states in ferroelectrics and sheds light into the potential of shear stress and strain engineering in control and design of ferroelectric devices based on exotic dipole states (including skyrmions).

\section{METHODS}

Effective Hamiltonian method

A first-principles derived effective Hamiltonian of $\left(\mathrm{Ba}, \mathrm{Sr}^{2}\right) \mathrm{TiO}_{3}$ solid solutions is used in our investigation. ${ }^{15,45}$ The total energy is written as a function of local modes $\mathbf{u}_{i}$, local displacements $\mathbf{v}_{i}$, which is related to the inhomogeneous strain $\eta_{t}$, homogeneous strain $\eta_{H}$, alloy species $\sigma_{i}$ (in value of +1 or -1 corresponding to the presence of a $\mathrm{Ba}$ or $\mathrm{Sr}$ atom) and 

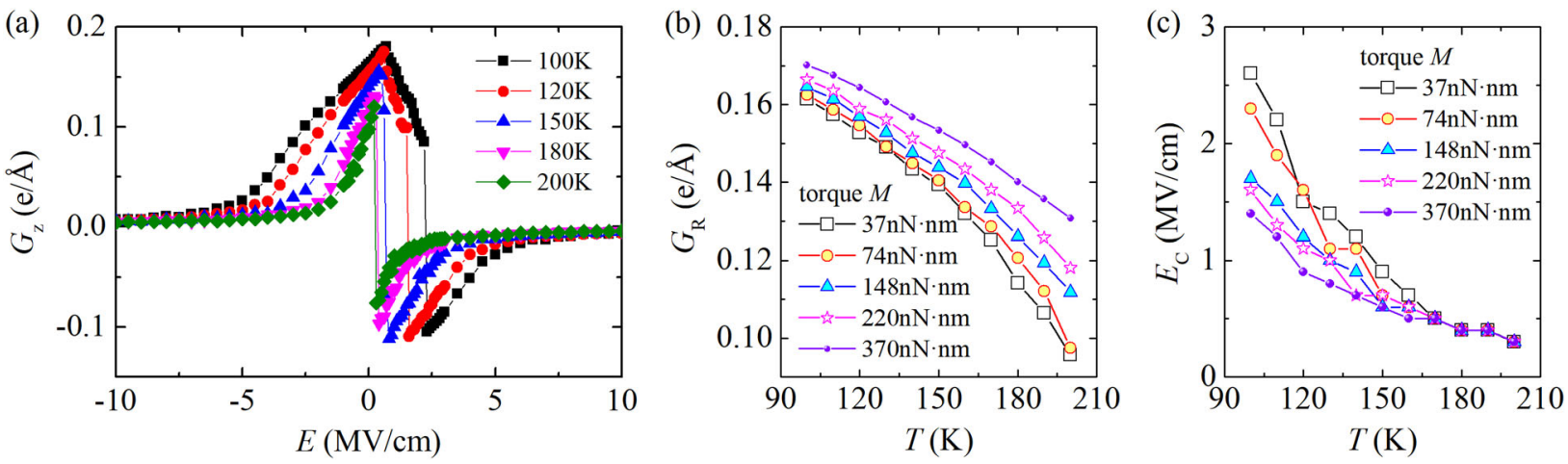

Fig. 6 Temperature dependence of the controllability of PTMO state by switching the axial polarization. a The calculated toroidization-electric field $\left(G_{z}-E\right)$ hysteresis loops with the torque $M$ being $70 \mathrm{nN} \mathrm{nm}$. b Remnant toroidization $G_{\mathrm{R}}$ and coercive force $E_{C}$ and under different temperatures and torques

the local strain $\eta_{\text {loc }}$ caused by the difference in ionic size between alloy species, i.e.,

$$
\begin{gathered}
E^{\text {tot }}\left(\left\{\mathbf{u}_{i}\right\},\left\{\mathbf{v}_{i}\right\},\left\{\eta_{\mathrm{H}, l}\right\},\left\{\sigma_{i}\right\},\left\{\eta_{\text {loc }}\right\}\right) \\
=E^{\text {ave }}\left(\left\{\mathbf{u}_{i}\right\},\left\{\mathbf{v}_{i}\right\},\left\{\eta_{\mathrm{H}, l}\right\}\right)+E^{\text {loc }}\left(\left\{\mathbf{u}_{i}\right\},\left\{\eta_{\mathrm{l}}\right\},\left\{\sigma_{i}\right\},\left\{\eta_{\text {loc }}\right\}\right)-\sum_{i} \mathbf{E} \cdot Z \mathbf{u}_{i}
\end{gathered},
$$

where $E^{\text {ave }}$ is the total energy associated with $\left(\mathrm{Ba}_{1} \mathrm{Sr}\right) \mathrm{TiO}_{3}$ system in the virtual crystal approximation (VCA), $E^{\text {loc }}$ gathers energy associated with alloying effects beyond the VCA, and the last term takes into account the effect of an external electric field $\mathbf{E}$ ( $Z$ is the Born effective charge). The explicit expression of $E^{\text {ave }}$ and $E^{\text {loc }}$ can be found in ref. ${ }^{45,46}$. The effect of applied twist force is modeled by adding the strain field induced by the twist force to the total strain of the nanowire. Such strain field can be numerically calculated by a finite element method based on the theory of elasticity (See Supplementary Information for details).

All the parameters of the effective Hamiltonian are determined by firstprinciples calculations. The effective Hamiltonian has been successfully applied to capture the ferroelectric properties of $\left(\mathrm{BaTiO}_{3}\right)_{m}\left(\mathrm{SrTiO}_{3}\right)_{n}$ systems. ${ }^{45}$ Surface effects of the systems are considered as a combination of the long-range and short-range contributions. The source of the longrange contribution is the depolarization field caused by the uncompensated polarization charges at the surfaces. The short-range contribution takes into account the polarization variations near the surfaces due to the short-range coupling of the atoms at the surface of the sample. For a nanowire, the bound charges at the lateral surfaces of the nanowire give rise to a strong depolarization field modifying the dipole states significantly. To consider this effect, an efficient dual-space approach based on periodic Green's function for dipole-dipole interaction in onedimensional periodic systems is adopted ${ }_{1}^{47}$ instead of the Ewald summation method for three-dimensional periodic systems. Moreover, the short-range interaction between the nanowire and the vacuum induces an additional surface energy, the explicit expression of which can be found in ref. ${ }^{48}$ Note, our previous study showed that the dipolar orders in $\left(\mathrm{BaTiO}_{3}\right)_{m}\left(\mathrm{SrTiO}_{3}\right)_{n}$ nanowires are not sensitive to the surface polarization when the size of the nanowire is not too small. ${ }^{15}$ This is due to the fact that toroidal order is mainly caused by the depolarization effect and it is a collective behavior. Therefore, the inclusion of a surface term would not alter the main physics of our results. For simplicity, the intrinsic surface energy term is turned off in our simulations.

The $\left(\mathrm{BaTiO}_{3}\right)_{m}\left(\mathrm{SrTiO}_{3}\right)_{n}$ nanowires considered in our simulations are rectangular shaped in lateral direction and are periodic along the longitudinal $z$-axis. The lengths of simulated supercells along $x$ - and $y$ axes are $L_{x}=n_{x} a_{0}$ and $L_{y}=n_{y} a_{0}$, respectively, while the $z$-axis periodic length $L_{z}$ is $L_{z}=(m+n) a_{0}$, with $a_{0}=3.9 \AA$ being the lattice constant of the primitive five-atom unit cell. Monte Carlo (MC) simulations are employed to combine with the first-principles derived effective Hamiltonian above to determine the dipole configuration of the model. An ideal open-circuit (OC) electrical boundary condition is imposed to the nanowire. The nanowires are firstly performed temperature annealing with a step of $10 \mathrm{~K}$ to obtain the initial PTMO state. External electric field along $z$-axis and twist force are then applied to the nanowires presenting PTMO state at given temperature to investigate the tunability of the PTMO state. At each situation, $40,000 \mathrm{MC}$ sweeps (the number can be up to $1,20,000$ in situations needed) are carried out to equilibrate the system and then
60,000 sweeps are used to get the statistical average. Theoretically, the order state of the PTMO state is characterized by the $z$-axial net polarization, i.e., $\mathbf{P}_{\mathbf{z}}=(V)^{-1} \sum_{i}^{N} \mathbf{p}_{i}$, and the toroidal order is characterized by toroidization $\mathbf{G}_{z}$, which is defined as $\mathbf{G}_{z}=(V)^{-1} \sum_{i}^{N} \mathbf{r}_{i} \times \delta \mathbf{p}_{i}$, where $\mathbf{r}_{i}$ is the position vector locating the $i$ unit cell and $\delta_{\mathbf{p} i}$ is the difference between the local dipole $\mathbf{p}_{i}$ and the averaged dipole, and the summation runs over all the $N$ unit cells of the system with the volume of the system being $V$.

\section{DATA AVAILABILITY}

The data that support the findings of this study are available from the corresponding author upon reasonable request.

\section{ACKNOWLEDGEMENTS}

This work was supported by the National Key Basic Research Program of China (Nos. 2015CB351905), NSFC (Nos. 11474363, 51172291, 11602310), and the Guangzhou Science and Technology Project (No. 201707020002). Y. Zheng also thanks support from the Special Program for Applied Research on Super Computation of the NSFCGuangdong Joint fund (the second phase), Fok Ying Tung Foundation, Guangdong Natural Science Funds for Distinguished Young Scholar and China Scholarship Council.

\section{AUTHOR CONTRIBUTIONS}

Y.Z. initiated and performed this work and manuscript. W.J.C. and Y.Z. conceived and designed the basic idea, structures. J.Y.L. performed the simulations. J.Y.L., W.J.C., Y.J., S.Y., L.L.D., and Y.Z. analyzed the results of simulations. J.Y.L., W.J.C., Y.J., S.Y., L.L.D., and Y.Z. co-write the manuscript. All authors contributed to discussion and reviewed the manuscript.

\section{ADDITIONAL INFORMATION}

Supplementary information accompanies the paper on the npj Computational Materials website (https://doi.org/10.1038/s41524-018-0135-2).

Competing interests: The authors declare no competing interests.

Publisher's note: Springer Nature remains neutral with regard to jurisdictional claims in published maps and institutional affiliations.

\section{REFERENCES}

1. Gacia, V. et al. Giant tunnel electroresistance for non-destructive readout of ferroelectric states. Nature 460, 81-84 (2009).

2. Luo, X., Wang, B. \& Zheng, Y. Tunable tunneling electroresistance in ferroelectric tunnel junctions by mechanical loads. ACS Nano 5, 1649-1656 (2011).

3. Fu, X., Naumov, I. I. \& Fu, H. Collective dipole behavior and unusual morphotropic phase boundary in ferroelectric $\mathrm{Pb}\left(\mathrm{Zr}_{0.5} \mathrm{Ti}_{0.5}\right) \mathrm{O}_{3}$ nanowires. Nano Lett. 13, 491-496 (2013). 
4. Scott, J. F. Applications of modern ferroelectrics. Science 315, 954-959 (2007).

5. Streiffer, S. K. et al. Observation of nanoscale $180^{\circ}$ stripe domains in ferroelectric $\mathrm{PbTiO}_{3}$ thin films. Phys. Rev. Lett. 89, 067601 (2002).

6. Stachiotti, M. G. Ferroelectricity in $\mathrm{BaTiO}_{3}$ nanoscopic structures. Appl. Phys. Lett. 84, 251-253 (2004).

7. Wu, Z., Duan, W., Huang, N., Wu, J. \& Gu, B.-L. Self-organization nanodomain structure in ferroelectric ultrathin films. Nanotechnology 18, 325703 (2007).

8. Chen, W. J. et al. Mechanical switching of ferroelectric domains beyond flexoelectricity. J. Mech. Phys. Solids 111, 43-66 (2018).

9. Kighelman, Z., Damjanovic, D., Cantoni, M. \& Setter, N. Properties of ferroelectric $\mathrm{PbTiO}_{3}$ thin films. J. Appl. Phys. 91, 1495-1501 (2002).

10. Choudhury, N., Walizer, L., Lisenkov, S. \& Bellaiche, L. Geometric frustration in compositionally modulated ferroelectrics. Nature 470, 513-517 (2011).

11. Naumov, I. I., Bellaiche, L. \& Fu, H. X. Unusual phase transitions in ferroelectric nanodisks and nanorods. Nature 432, 737-740 (2004)

12. Fu, H. \& Bellaiche, L. Ferroelectricity in barium titanate quantum dots and wires. Phys. Rev. Lett. 91, 257601 (2003).

13. Yadav, A. K. et al. Observation of polar vortices in oxide superlattices. Nature 530, 198-201 (2016).

14. Louis, L., Kornev, I., Geneste, G., Dkhil, B. \& Bellaiche, L. Novel complex phenomena in ferroelectric nanocomposites. J. Phys. 24, 402201 (2012).

15. Chen, W. J., Zheng, Y. \& Wang, B. Large and tunable polar-toroidal coupling in ferroelectric composite nanowires toward superior electromechanical responses. Sci. Rep. 5, 11165 (2015)

16. Nahas, Y. et al. Discovery of stable skyrmionic state in ferroelectric nanocomposites. Nat. Commun. 6, 8542 (2015).

17. Prosandeev, S., Ponomareva, I., Kornev, I. \& Bellaiche, L. Control of vortices by homogeneous fields in asymmetric ferroelectric and ferromagnetic rings. Phys. Rev. Lett. 100, 047201 (2008).

18. Chen, W. J. \& Zheng, Y. Vortex switching in ferroelectric nanodots and its feasibility by a homogeneous electric field: effects of substrate, dislocations and local clamping force. Acta Mater. 88, 41-54 (2015).

19. Yuan, S. et al. Defect-mediated vortex multiplication and annihilation in ferroelectrics and the feasibility of vortex switching by stress. Acta Mater. 148, 330-343 (2018).

20. Chen, W. J., Zheng, Y. \& Wang, B. Pinning effects of dislocations on vortex domain structure in ferroelectric nanodots. Appl. Phys. Lett. 104, 222912 (2014).

21. Prosandeev, S., Ponomareva, I., Kornev, I., Naumov, I. \& Bellaiche, L. Controlling toroidal moment by means of an inhomogeneous static field: an ab initio study. Phys. Rev. Lett. 96, 237601 (2006).

22. Ma, L. L. et al. Direct electrical switching of ferroelectric vortices by a sweeping biased tip. Acta Mater. (in the press).

23. Hong, Z. \& Chen, L.-Q. Blowing polar skyrmion bubbles in oxide superlattices. Acta Mater. 152, 155-161 (2018).

24. Prosandeev, S. \& Bellaiche, L. Properties of ferroelectric nanodots embedded in a polarizable medium: atomistic simulations. Phys. Rev. Lett. 97, 167601 (2006).

25. Chen, W. J., Zheng, Y., Wang, B. \& Liu, J. Y. Coexistence of toroidal and polar domains in ferroelectric systems: a strategy for switching ferroelectric vortex. J. Appl. Phys. 115, 214106 (2014).

26. Gui, Z. \& Bellaiche, L. Terahertz dynamics of ferroelectric vortices from first principles. Phys. Rev. B 89, 064303 (2014).

27. Naumov, I. \& Fu, H. Vortex-to-polarization phase transformation path in ferroelectric $\mathrm{Pb}(\mathrm{ZrTi}) \mathrm{O}_{3}$ nanoparticles. Phys. Rev. Lett. 98, 077603 (2007)

28. Chen, W. J. et al. Mechanical switching in ferroelectrics by shear stress and its implications on charged domain wall generation and vortex memory devices. RSC Adv. 8, 4434-4444 (2018).

29. Tian, $\mathrm{G}$. et al. Magnetoelectric coupling in well-ordered epitaxial $\mathrm{BiFeO}_{3} / \mathrm{CoFe}_{2} \mathrm{O}_{4} /$ $\mathrm{SrRuO}_{3}$ heterostructure nanodot array. ACS Nano 10, 1025-1032 (2016).
30. Lu, X. et al. Magnetoelectric coupling in ordered arrays of multilayered heteroepitaxial $\mathrm{BaTiO}_{3} / \mathrm{CoFe}_{2} \mathrm{O}_{4}$ nanodots. Nano Lett. 11, 3202-3206 (2011).

31. Lim, S. K., Crawford, S., Haberfehlner, G. \& Gradečak, S. Controlled modulation of diameter and composition along individual III-V nitride nanowires. Nano Lett. 13, 331-336 (2012).

32. Schilling, A., Bowman, R. M., Catalan, G., Scott, J. F. \& Gregg, J. M. Morphological control of polar orientation in single-crystal ferroelectric nanowires. Nano Lett. 7, 3787-3791 (2007).

33. Urban, J. J., Spanier, J. E., Ouyang, L., Yun, W. S. \& Park, H. Single-crystalline barium titanate nanowires. Adv. Mater. 15, 423-426 (2003).

34. Urban, J. J., Yun, W. S., Gu, Q. \& Park, H. Synthesis of single-crystalline perovskite nanorods composed of barium titanate and strontium titante. J. Am. Chem. Soc. 124, 1186-1187 (2002).

35. Mao, Y., Banerjee, S. \& Wong, S. Large-scale synthesis of single-crystalline perovskite nanostructures. J. Am. Chem. Soc. 125, 15718-15719 (2003).

36. Yuan, S. et al. Torsion-induced vortex switching and skyrmion-like state in ferroelectric nanodisks. J. Phys. 30, 465304 (2018).

37. Srivastava, S. et al. Light-controlled self-assembly of semiconductor nanoparticles into twisted ribbons. Science 327, 1355-1359 (2010).

38. Gao, Z. et al. Effects of piezoelectric potential on the transport characteristics of metal-ZnO nanowire-metal field effect transistor. J. Appl. Phys. 105, 113707 (2009).

39. Bierman, M. J., Lau, Y. K. A., Kvit, A. V., Schmitt, A. L. \& Jin, S. Dislocation-driven nanowire growth and Eshelby twist. Science 320, 1060-1063 (2008).

40. Wang, Z. L. \& Song, J. Piezoelectric nanogenerators based on zinc oxide nanowire arrays. Science 312, 242-246 (2006).

41. Stan, G., Ciobanu, C. V., Parthangal, P. M. \& Cook, R. F. Diameter-dependent radial and tangential elastic moduli of ZnO nanowires. Nano Lett. 7, 3691-3697 (2007).

42. Yoo, J. et al. Effects of mechanical deformation on energy conversion efficiency of piezoelectric nanogenerators. Nanotechnology 26, 275402 (2015).

43. Wu, B., Heidelberg, A. \& Boland, J. J. Mechanical properties of ultrahigh-strength gold nanowires. Nature 4, 525-529 (2005).

44. Mølhvae, K., Wich, T., Kortschack, A. \& Bøggild, P. Pick-and-place nanomanipulation using microfabricated grippers. Nanotechnology 17, 2434-2441 (2006).

45. Walizer, L., Lisenkov, S. \& Bellaiche, L. Finite-temperature properties of $\left(\mathrm{Ba}, \mathrm{Sr}^{2} \mathrm{TiO}_{3}\right.$ systems from atomistic simulations. Phys. Rev. B 73, 144105 (2006).

46. Zhong, W., Vanderbilt, D. \& Rabe, K. M. First-principles theory of ferroelectric phase transitions for perovskites: the case of $\mathrm{BaTiO}_{3}$. Phys. Rev. B 52, 6301 (1995).

47. Naumov, I. I. \& Fu, H. A new method for determining dipole-dipole energy in 1D and 2D systems. arXiv:cond-mat/0505497v2 (2005).

48. Almahmoud, E., Navtsenya, Y., Kornev, I., Fu, H. \& Bellaiche, L. Properties of $\mathrm{Pb}(\mathrm{Zr}$, $\mathrm{Ti}_{3} \mathrm{O}_{3}$ ultrathin films under stress-free and open-circuit electrical boundary conditions. Phys. Rev. B 70, 220102 (2004).

Open Access This article is licensed under a Creative Commons Attribution 4.0 International License, which permits use, sharing, adaptation, distribution and reproduction in any medium or format, as long as you give appropriate credit to the original author(s) and the source, provide a link to the Creative Commons license, and indicate if changes were made. The images or other third party material in this article are included in the article's Creative Commons license, unless indicated otherwise in a credit line to the material. If material is not included in the article's Creative Commons license and your intended use is not permitted by statutory regulation or exceeds the permitted use, you will need to obtain permission directly from the copyright holder. To view a copy of this license, visit http://creativecommons. org/licenses/by/4.0/.

(c) The Author(s) 2018 